\title{
A possible variant of negative motor seizure arising from the supplementary negative motor area.
}

\section{$\operatorname{AUTHOR}(S):$}

Yamao, Yukihiro; Matsumoto, Riki; Kunieda, Takeharu; Arakawa, Yoshiki; Kikuchi, Takayuki; Shibata, Sumiya; Shimotake, Akihiro; Fukuyama, Hidenao; Ikeda, Akio; Miyamoto, Susumu

\section{CITATION:}

Yamao, Yukihiro ...[et al]. A possible variant of negative motor seizure arising from the supplementary negative motor area.. Clinical neurology and neurosurgery 2015, 134: 126129

\section{ISSUE DATE:}

2015-07

URL:

http://hdl.handle.net/2433/210374

\section{RIGHT:}

(c) 2015. This manuscript version is made available under the CC-BY-NC-ND 4 . 0 license

http://creativecommons.org/licenses/by-nc-nd/4.0/; The full-text file will be made open to the public on 1 July 2016 in accordance with publisher's 'Terms and Conditions for Self-Archiving'.; This is not the published version. Please cite only the published version.; この論文は出版社版でありません。引用の際には出版社版をご確認ご利用ください。 
A possible variant of negative motor seizure arising from the supplementary

negative motor area

Yukihiro Yamao a, Riki Matsumoto b,*, Takeharu Kunieda a,*, Yoshiki Arakawa a,

Takayuki Kikuchi ${ }^{\text {a }}$, Sumiya Shibata ${ }^{a}$, Akihiro Shimotake ${ }^{\mathrm{c}}$, Hidenao Fukuyama ${ }^{\mathrm{d}}$,

Akio Ikeda $^{\mathrm{b}}$, and Susumu Miyamoto ${ }^{\mathrm{a}}$

${ }^{\text {a }}$ Department of Neurosurgery, Kyoto University Graduate School of Medicine

${ }^{\mathrm{b}}$ Department of Epilepsy, Movement Disorders and Physiology, Kyoto University

Graduate School of Medicine

${ }^{\text {c }}$ Department of Neurology, Kyoto University Graduate School of Medicine

${ }^{d}$ Human Brain Research Center, Kyoto University Graduate School of Medicine

Corresponding to: Riki Matsumoto \& Takeharu Kunieda,

Kyoto University Graduate School of Medicine, Kyoto, Japan

54,Shogoin Kawahara-cho, Sakyo-ku, Kyoto, 606-8507, Japan 
tel \& fax: +81-75-751-3772 \& +81-75-751-9416

email: matsumot@kuhp.kyoto-u.ac.jp (RM), kuny@kuhp.kyoto-u.ac.jp (TK)

Key words: negative motor area, motor evoked potential, ictal onset zone

\section{Abbreviations}

NMS: negative motor seizure, NMA: negative motor area, SMA: supplementary motor area, VAC: vertical anterior commissure, EEG: electroencephalogram, ECoG:

electrocorticogram, MEP: motor evoked potentials 
Yamao et al. 3

\section{Introduction}

High-frequency electrical cortical stimulation of a certain cortical area in the frontal

lobe produced “negative effects”, arrest of voluntary movements without lack of

consciousness; this cortical area has been termed "negative motor area (NMA)” [1, 2].

Negative motor seizure (NMS) is a rare seizure that involves only the inability to

conduct voluntary movements or praxis, without lack of consciousness [3]. To our

knowledge, in only one report [3], by analyzing the features common to three cases,

NMS were found to originate within the broad lateral and medial areas defined as NMA,

but the specific electrodes showing the seizure-onset zone did not necessarily produce

negative motor responses. NMA seems to be responsible for NMS, but the precise

origin and mechanism of NMS has not been fully clarified.

Here we report a patient with a tumor in the medial right frontal lobe who

manifested NMS as the sole seizure semiology, and who became seizure-free after

resection of the tumor and adjacent NMA.

\section{Case report}


Yamao et al. 4

The patient was a 34-year-old, right-handed man. One year prior to referral to Kyoto

University Hospital, he began experiencing brief stereotyped episodes in which he felt

mirth without laughter for a few seconds while reading comic books whose contents

were not funny. Six months later, he began having difficulty or slowing for a few

seconds at a time while using both arms (e.g., while cleaning his body with a towel) or

speaking in front of an audience, though others did not notice. None of these events

devolved into positive motor seizures. Because the stereotyped episodes occurred

repeatedly (once or twice a month), he consulted a neurosurgeon at his local hospital.

His brain MRI revealed a tumor in the medial right frontal area, and the patient was

referred to our hospital. Neurological examination was unremarkable, including lack of

difficulty with alternating hand movements, which is typically seen in supplementary

motor area (SMA) syndrome. MRI showed a T1-hypo-, T2-hyper-intensity lesion

without gadolinium enhancement in the medial right frontal lobe anterior to the vertical

anterior commissure (VAC) line (Fig. 1A). NMS in association with a low-grade tumor

was suspected and 3-days video-electroencephalogram (EEG) monitoring with scalp

electrodes was performed. No habitual seizures were detected, and only a few sharp 
Yamao et al. 5

transients were recorded interictally at the right fronto-central area with a maximum at

C4 (Fig. 1B).

The patient underwent awake craniotomy to probe motor function and identify a possible epileptic focus at or around the tumor. Two $4 \times 5$ subdural grid electrodes (recording diameter of $3 \mathrm{~mm}$, inter-electrode distance $1 \mathrm{~cm}$, Unique Medical, Tokyo, Japan) were placed over both the lateral and medial sides of the right frontal lobe. A 32-channel intraoperative monitoring system (MEE 1232 Neuromaster \& MS 120B, Nihon-Koden, Tokyo, Japan) was used both for recording raw electrocorticogram (ECoG) data and delivering electrical stimuli. ECoG data was digitized with a sampling rate of $5,000 \mathrm{~Hz}$ and a band-pass filter of $0.5-1500 \mathrm{~Hz}$.

To avoid the risk of evoking seizures, functional motor mapping was performed in the awake state by applying single-pulse electrical cortical stimulation (pulse width of $0.3 \mathrm{~ms}, 1 \mathrm{~Hz}$, alternating polarity, 10-15 mA) and recording motor evoked potentials (MEPs), as reported elsewhere [4]. Surface electromyograms were recorded from the bilateral deltoid, extensor carpi radialis, abductor pollicis brevis and tibialis anterior muscles. MEPs were recorded in the resting condition and during 
Yamao et al. 6

adequate muscle contractions at the same stimulus intensity. In electrodes where MEPs

were not elicited by 1-Hz electrical cortical stimulation, 5-train electrical cortical

stimulation (a square pulse of $0.2 \mathrm{~ms}$ with $2 \mathrm{~ms}$ interval, 10-15 mA) was applied to

identify motor function [5]. Functional motor mapping was performed only for the

clinical purpose, and the electrical cortical stimulation was approved by the Kyoto

University Graduate School and Faculty of Medicine, Ethics Committee (IRB C573).

Since we had not performed intraoperative MRI, the placement of electrodes was

identified based on operative observation and neuro-navigation data.

In the awake state, frequent interictal spikes were focally recorded posterior to the tumor in the medial frontal area (Electrodes B09, 13, 14, and 19 in Fig. 2 and 3A).

Spikes occurred most frequently at Electrode B09. No spikes were shown on the lateral frontal cortex. Based on MEP patterns elicited by 1-Hz electrical cortical stimulation as defined previously [4] (e.g., the response of the bilateral upper extremities or contralateral lower extremity), the SMA was defined at the electrode pairs of B01-02, B06-07, and B12-17, and the left leg region of the primary motor area at B11-16 (Fig. 3B). At B08-09 neither 1-Hz nor 5-train electrical cortical stimulation elicited MEPs 
Yamao et al. 7

even with muscle contraction, but only 5-train electrical cortical stimulation, which

delivers more intense electrical charges, produced a negative motor response, that is, a short lapse of posture in the bilateral arms. Judging from its location around the VAC line and rostral to the hand SMA, this area was regarded as the supplementary NMA [3].

After the removal of the tumor and cortices where interictal spikes occurred during awake surgery (dotted line circle in Fig. 3), the patient developed left hemiparesis that fully resolved within 5 weeks. He has been seizure- or event-free for more than 24 months postoperatively. The tumor pathology was diffuse astrocytoma (WHO grade II).

\section{Discussion}

The semiology of NMS has been reported to include (1) indescribable or ill-localized aura, (2) repetitive involuntary vocalization, (3) inability to speak, (4) inability to move the extremities, and (5) subsequent evolution to positive motor seizures in a conscious state [3]. NMS differs from focal inhibitory seizure or ictal monoparesis, whose seizures 
Yamao et al. 8

are characterized by paresis or paralysis of extremities and whose symptomatogenic

zone is the primary sensorimotor area. NMA is supposedly responsible for NMS, and the supplementary NMA is located between the pre-SMA and the border of the VAC line of the SMA [3].

In ECoG, interictal spikes were most frequently recorded at Electrode B08, which corresponded with the supplementary NMA, based on 1-Hz electrical cortical stimulation (absence of MEPs) and 5-train electrical cortical stimulation (a short lapse of posture in the bilateral arms). In previous electrical stimulation studies, stimulation of supplementary NMA did not elicited MEPs [5], which is compatible with the present findings. Because the patient's symptoms were mild and continued only for a few seconds, seizures were neither noticed nor videotaped at home. In addition, habitual seizures were not recorded with video-EEG monitoring. Therefore, the limitation leaves some degree of uncertainty with regards to the diagnosis of his seizures. We could not completely exclude the non-epileptic seizures such as cataplexy. His reported semiology, i.e., stereotyped slowing/difficulty of bimanual movements or speech without loss of awareness, suggests the diagnosis of a variant of NMS. Indeed, this diagnosis is further 
Yamao et al. 9

supported by the resolution of these events after resection of the tumor and a small

adjacent cortical region at and around the supplementary NMA where frequent interictal spikes were recorded in ECoG. Tight connections have been reported between the medial and lateral premotor areas by means of cortico-cortical evoked potentials [6]. In our case, no spikes were recorded from the lateral premotor area despite frequent focal spikes in of the medial premotor area. Taken together with the ictal negative symptoms that was milder than previously reported [3], e.g., mild, brief slowing of speech or bimanual movements and lack of evolution to positive motor seizures, it is likely that the epileptic activity was restricted to a very focal region at and around the supplementary NMA. Although we could not record ictal ECoG patterns with chronic electrode implantation, it is likely that the symptomatogenic zone and probably the ictal onset zone were located at the supplementary NMA. The initial aura of mirth also supports this hypothesis since, in a previous study [7], 50-Hz electrical stimulation of the cortex just anterior to the VAC line elicited mirth. In a previous report [8], by using ECoG, the resection of seizure foci adding to the tumor resection got better seizure control. In our case, because the ictal onset zone was probably resected, he has been 
Yamao et al. 10

seizure-free for more than 24 months. Further case accumulation is warranted to

establish the supplementary NMA as one of the symptomatogenic zones of NMS.

\section{Disclosure of Interest}

None of the authors has any conflict of interest to disclose.

\section{Acknowledgements}

This work was partly supported by Grants-in-Aid for Scientific Research (KAKENHI)

(B) 26282218 (RM), (C) 24592159 (TK), Grants-in-Aid for Young Scientists (B)

25861273 (TK), and Exploratory Research 26560465 (RM) from the Ministry of

Education, Culture, Sports, Science and Technology of Japan. Department of Epilepsy,

Movement Disorders and Physiology, Kyoto University Graduate School of Medicine is an endowment department, supported with grants by GlaxoSmithKline K.K., NIHON

KOHDEN CORPORATION, Otsuka Pharmaceutical Co., and UCB Japan Co., Ltd. 
Yamao et al. 11

\section{References}

1. Lüders HO, Dinner DS, Morris HH, Wyllie E, Comair YG. Cortical electrical stimulation in humans. The negative motor areas. Advances in neurology.

1995;67:115-129.

2. Filevich E, Kuhn S, Haggard P. Negative motor phenomena in cortical stimulation: implications for inhibitory control of human action. Cortex.

2012;48:1251-1261.

3. Ikeda A, Hirasawa K, Kinoshita M, Hitomi T, Matsumoto R, Mitsueda T, Taki JY, Inouch M, Mikuni N, Hori T, Fukuyama H, Hashimoto N, Shibasaki H, Takahashi R. Negative motor seizure arising from the negative motor area: is it ictal apraxia? Epilepsia. 2009;50:2072-2084.

4. Kikuchi T, Matsumoto R, Mikuni N, Yokoyama Y, Matsumoto A, Ikeda A, Fukuyama H, Miyamoto S, Hashimoto N. Asymmetric bilateral effect of the supplementary motor area proper in the human motor system. Clinical neurophysiology. 2012;123:324-334.

5. Usui N, Terada K, Baba K, Matsuda K, Tottori T, Umeoka S, Mihara T, 
Nakamura F, Usui K, Inoue Y. Extraoperative functional mapping of motor areas in

epileptic patients by high-frequency cortical stimulation. J Neurosurg.

2008;109:605-614.

6. Matsumoto R, Nair DR, LaPresto E, Bingaman W, Shibasaki H, Lüders HO.

Functional connectivity in human cortical motor system: a cortico-cortical evoked potential study. Brain. 2007;130:181-197.

7. Krolak-Salmon P, Hénaff MA, Vighetto A, Bauchet F, Bertrand O, Mauguière F, Isnard J. Experiencing and detecting happiness in humans: the role of the supplementary motor area. Ann Neurol. 2006;59:196-199.

8. Berger MS, Ghatan S, Haglund MM, Dobbins J, Ojemann GA. Low-grade gliomas associated with intractable epilepsy: seizure outcome utilizing electrocorticography during tumor resection. J Neurosurg. 1993;79:62-69. 


\section{Figure legends}

\section{Fig. 1}

Preoperative MRI data (A) and interictral electroencephalogram with scalp electrodes

(B). A preoperative MRI shows a T1-hypo-, T2-hyper-intensity (A: left) tumor without gadolinium enhancement (A: middle and right) in the medial right frontal lobe anterior to the vertical anterior commissure line. Only a sharp transient is shown at the right fronto-central area with a maximum at C4 (B). AC-PC line: anterior commissure-posterior commissure line, VAC: vertical anterior commissure, VPC: vertical posterior commissure, ECG: electrocardiogram

\section{Fig. 2}

Interoperative electrocorticogram with subdural electrodes. Interictal spikes are shown at Electrodes B09, B13, B14, and B19. The most frequent spikes occur at Electrode B09. At Electrode B16, $\mu$ waves are recorded. All subdural electrodes were referenced to a scalp electrode placed on the skin over the left mastoid process. 
Yamao et al. 14

Fig. 3

The schema of interictal spikes (A) and cortical mapping (B). Subdural grid electrodes were placed on the right medial frontal cortices caudal to the tumor. Positive cortical motor functions based on MEPs elicited by 1-Hz electrical cortical stimulation are shown. Only 5-train electrical cortical stimulation produced a negative motor response (B08-09). The removal area was determined by coregistration with the postoperative MRI. Details of motor mapping using 5-train electrical cortical stimulation will be reported elsewhere for a different purpose. CS: central sulcus, MEP: motor evoked potential, AC-PC line: anterior commissure-posterior commissure line, VAC: vertical anterior commissure, VPC: vertical posterior commissure. 
Fig. 2

B01

B02

B03

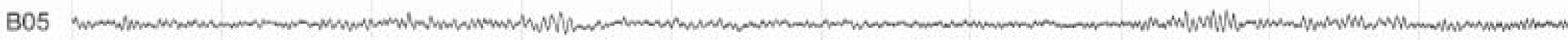

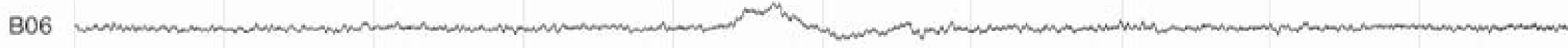

BO7 marmanm

B08 208



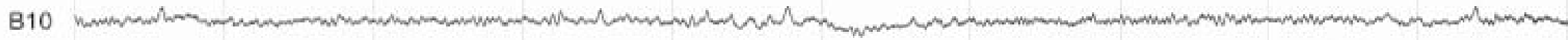

B11

B12 Whotum

B13 raphom

B14

B15



B17 Wr.

B19

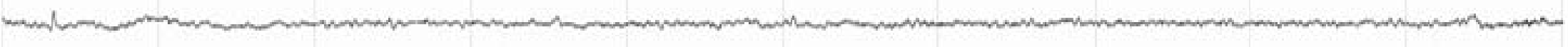

$\mathrm{B} 20$

" $\mu$ wave

$300 \mu \mathrm{V}$ 
